\section{DESIGN OF COUPLING STRUCTURES FOR GROOVE GUIDE RESONATORS}

Thomas F. Bechteler, ${ }^{1}$ Alp Kustepeli, ${ }^{2}$ and

Sevinc A. Bechteler ${ }^{2}$

${ }_{1}^{1}$ Radar Department, MilSon Ltd., Gaziemir-Aegean Free Zone, 35410 Izmir, Turkey; Corresponding author:

thomas.bechteler@gmx.de

2 Department of Electrical-Electronics Engineering, Izmir Institute of

Technology, 35430 Izmir, Turkey

\section{Received 24 September 2007}

ABSTRACT: Three different coupling structures for a groove guide resonator operating at $10 \mathrm{GHz}$ are investigated. First, propagatable modes are determined analytically. Then, the efficiency of the coupling structures is investigated by means of the method of moments and measurements. Finally, a groove guide resonator is designed and the resonance spectrum for each coupling structure is computed numerically, applying the finite-difference time-domain method. Numerical results are compared with experimental measurements. There is good agreement between the measured and simulated data. (c) 2008 Wiley Periodicals, Inc. Microwave Opt Technol Lett 50: 1406-1410, 2008; Published online in Wiley InterScience (www.interscience.wiley.com). DOI 10.1002/ mop. 23372

Key words: groove guide; resonator; coupling structure; finite-difference time-domain; method of moments

\section{INTRODUCTION}

When the groove guide was presented in 1963 by Tischer [1], its low-loss property and the fact that it is an open structure led to several applications. In [2], the groove guide as a leaky waveguide for railway communications was proposed. Groove guide ring resonators were proposed and analyzed in [3, 4]. A system to measure dielectric properties at microwaves was presented in [5]. Furthermore, a groove guide leaky-wave antenna was characterized in [6], and another wide-band antenna application using a circular groove guide structure was analyzed in [7].

(a)

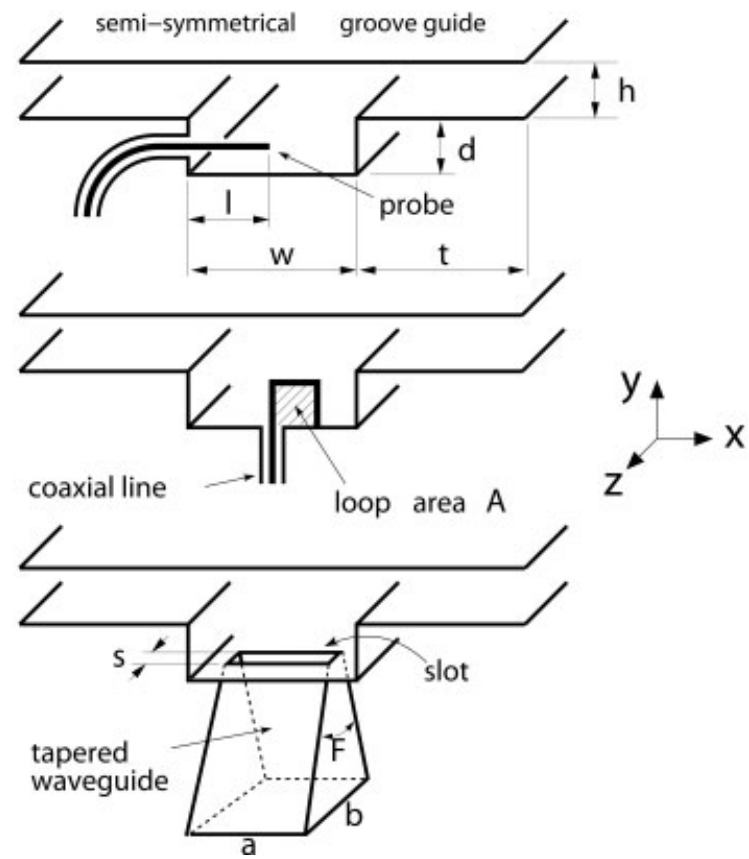

Figure 1 (a) Probe length $l$, (b) Loop area $A$ and (c) Slot width $s$

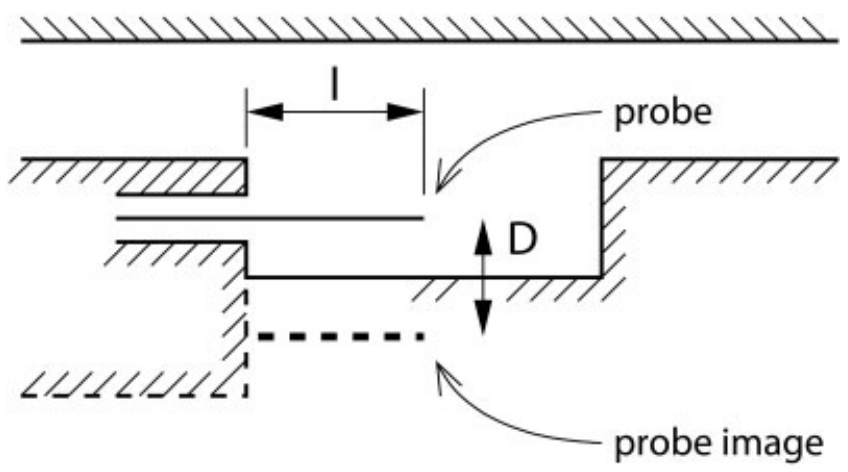

Figure 2 Probe of length $l$ with image

In the past, scant attention was paid to coupling structures for groove guides. In [8], the so-called mode launcher has been proposed. The mode launcher requires that one end of the groove guide be accessible. In some applications, e.g. resonator structures, this is not the case. In [9], other possible coupling structures were investigated by means of the finite-difference time-domain (FDTD) method.

In this work, the coupling structures for a groove guide resonator are investigated numerically and experimentally, and optimum dimensions are found. A groove guide resonator including the coupling structures is fabricated and measured.

\section{DESIGN CRITERIA FOR COUPLING STRUCTURES}

To investigate the frequency behavior of coupling structures, a probe, loop, and slot are placed inside a groove guide as shown in Figure 1 . The physical dimensions of the groove guide are given as groove width $w=30 \mathrm{~mm}$, groove depth $d=10 \mathrm{~mm}$, plate distance $h=10 \mathrm{~mm}$, and outer region length $t=60 \mathrm{~mm}$. Wave propagation is along the $z$-axis.

The cut-off frequencies of the groove guide can be calculated with the given dimensions. The method after Fernyhough and Evans [10] yields the modes and their cut-off frequencies which can propagate within the $\mathrm{X}$-band $(8-12 \mathrm{GHz})$ as

$$
\mathrm{TE}_{11}: 7.79 \mathrm{GHz} ; \quad \mathrm{TM}_{11}: 8.78 \mathrm{GHz} ; \quad \mathrm{TE}_{21}: 9.55 \mathrm{GHz},
$$

where the first index indicates the field variation in the $x$-direction and the second index the field variation in the $y$-direction. As already predicted in [9], the probe and the loop will excite the $\mathrm{TM}_{11}$-mode and $\mathrm{TE}_{11}$-mode, respectively. The slot in this work is oriented in such a way that it will excite the $\mathrm{TM}_{11}$-mode.

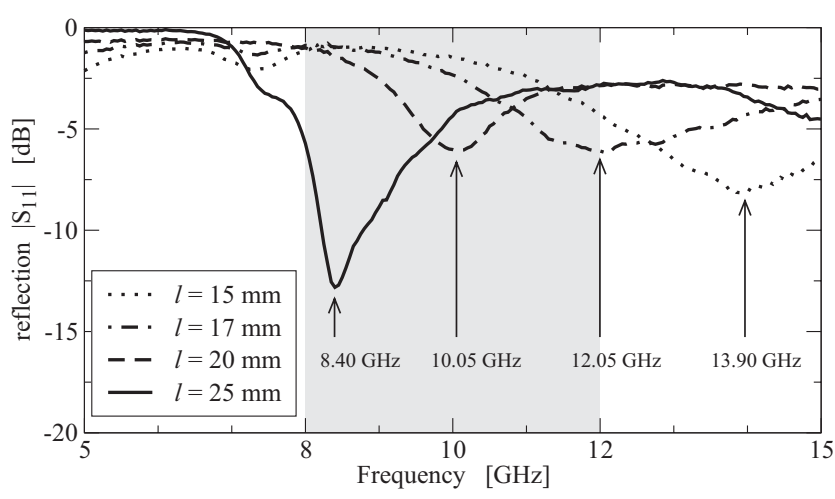

Figure $3|S 11|$ measurement of probe inside the groove depending on $l$ 
TABLE 1 Computed and Measured Resonances for the Probe

\begin{tabular}{lccc}
\hline & \multicolumn{3}{c}{ Probe Resonances in GHz } \\
\cline { 2 - 4 } $\begin{array}{l}\text { Probe } \\
\text { Length } l\end{array}$ & $\begin{array}{c}\text { Coupled Probes } \\
\text { on Infinite } \\
\text { Ground MoM }\end{array}$ & $\begin{array}{c}\text { Coupled Probes on } \\
\text { Infinite Ground } \\
\text { Eqs. (2) and (3) }\end{array}$ & $\begin{array}{c}\text { Probe Inside } \\
\text { Groove } \\
\text { Measurement }\end{array}$ \\
\hline $15 \mathrm{~mm}$ & 13.96 & 13.72 & 13.90 \\
$17 \mathrm{~mm}$ & 12.38 & 11.99 & 12.05 \\
$20 \mathrm{~mm}$ & 10.58 & 9.79 & 10.05 \\
$25 \mathrm{~mm}$ & 8.56 & 8.44 & 8.40 \\
\hline
\end{tabular}

\subsection{Probe Coupling}

The probe can be considered as a monopole radiating into the groove guide. Since the probe is inside the groove and, therefore, close to a metallic wall, two coupled probes have to be considered using the method of images, to have an idea about the resonances. The resonant frequency shifts because of the mutual coupling between the probe and its image. Figure 2 depicts the probe inside the groove guide together with its image, whereas their distance is $D=10 \mathrm{~mm}$.

Since the currents in the probe and its image are of same magnitude but of opposite direction, the input impedance of the probe is written as

$$
\mathrm{Z}_{\text {in }}=\mathrm{Z}_{11}-\mathrm{Z}_{12}=\left(R_{11}-R_{12}\right)+j\left(X_{11}-X_{12}\right)
$$

where $Z_{11}$ is the self impedance and $Z_{12}$ is the mutual impedance. Resonances occur where the imaginary part of Zin becomes zero. The input impedance has been computed based on the induced emf method presented in $[11,12]$. For probe lengths much larger than the probe radius, the induced emf method yields close results to the approaches presented in $[13,14]$ which make use of the Poynting's vector theorem and integral equation method, respectively. Hence, the induced emf method yields accurate results and a closed form for the input impedance. The imaginary part of $Z_{\text {in }}$ is

$$
\begin{array}{r}
X_{11}=\frac{1}{2} \cdot \frac{\sqrt{\varepsilon_{0} / \mu_{0}}}{4 \pi \sin ^{2}(\beta l / 2)}\{2 \operatorname{Si}(\beta l)+\cos (\beta l)[2 \operatorname{Si}(\beta l)-\operatorname{Si}(2 \beta l)] \\
\left.-\sin (\beta l)\left[2 \operatorname{Ci}(\beta l)-2 \operatorname{Ci}(2 \beta l)-\operatorname{Ci}\left(\frac{2 \beta r^{2}}{l}\right)\right]\right\}
\end{array}
$$

and

$$
\begin{array}{r}
X_{12}=\frac{1}{2} \cdot \frac{\sqrt{\varepsilon_{0} / \mu_{0}}}{4 \pi \sin ^{2}(\beta l / 2)}\{-2[2+\cos (\beta l)] \cdot \operatorname{Si}(\beta D) \\
+4 \cos ^{2}(\beta l / 2)\left[\operatorname{Si}\left(\beta u^{\prime} / 2\right)+\operatorname{Si}(\beta u / 2)\right]
\end{array}
$$

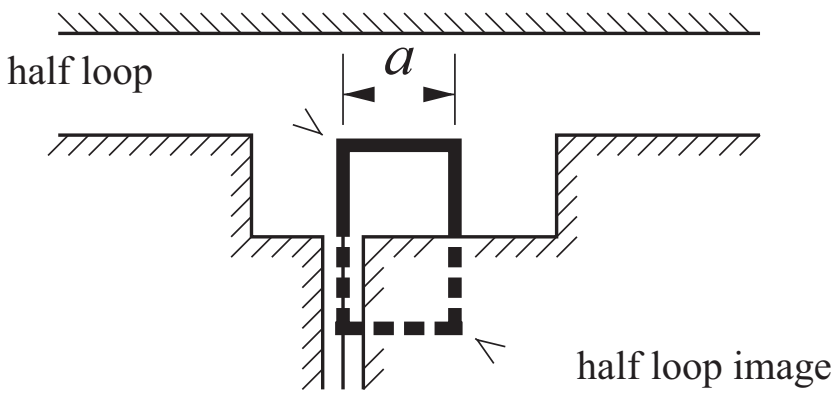

Figure 4 Loop with side length $a$

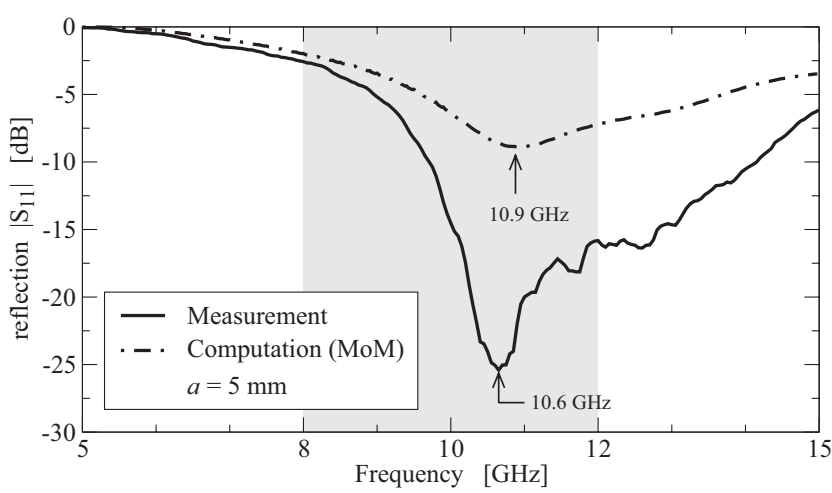

Figure 5 Computed and measured $|S 11(f)|$ of loop: $a=5 \mathrm{~mm}$

$$
\begin{gathered}
-\cos (\beta l)\left[\operatorname{Si}\left(\beta v^{\prime}\right)+\operatorname{Si}(2 \beta v)\right] \\
\left.+\sin (\beta l)\left[\operatorname{Ci}(\beta v)-\operatorname{Ci}\left(\beta v^{\prime}\right) b f-2 \operatorname{Ci}(\beta u / 2)+2 \operatorname{Ci}\left(\beta u^{\prime} / 2\right)\right]\right\}
\end{gathered}
$$

with $u=\sqrt{4 D^{2}+l^{2}}+l, u^{\prime}=\sqrt{4 D^{2}+l^{2}}-l, v=\sqrt{ } D^{2}+$ $l^{2}+l, v^{\prime}=\sqrt{D^{2}+l^{2}}-l, \mu_{0}=1.257 \cdot 10^{-6} \mathrm{Vs} / \mathrm{Am}$, and $\varepsilon_{0}$ $=8.854 \cdot 10^{-12} A s / V m$, where $D$ is the distance between the probe and its image, $l$ and $r$ are the probe length and radius, respectively, $\beta=2 \pi f / c_{0}$ is the propagation constant in vacuum, and $\operatorname{Si}(x)$ and $\mathrm{Ci}(x)$ are the sine and cosine integrals [15], respectively. Solving $X_{11}-X_{12}=0$ with respect to $f$ yields the resonance frequencies of the probe in free space. Figure 3 shows the measured frequency response of the reflection $S_{11}$ for four different probe lengths $l$ and the corresponding resonance frequencies. The radius $r$ of the used probes is $0.4 \mathrm{~mm}$ for all cases. For comparison, two coupled probes on an infinite ground plane have been also computed by means of the Method of Moments (MoM) [16]. The computed resonances in free space together with the measured resonances in the groove for four different probe lengths are listed in Table 1. All three results are in good agreement and, therefore, the probe length can be assessed by using Eqs. (2) and (3), although, the measured probe is inside a groove structure and not on infinite ground. To excite the groove guide in the X-band, the probe length must be chosen between 17 and $25 \mathrm{~mm}$. For a probe resonance about 9 $\mathrm{GHz}$, the cutoff frequency of the TM11-mode, the length was optimized to $l=24 \mathrm{~mm}$.

\subsection{Loop Coupling}

The loop used to excite the groove guide is shown in Figure 4. It can be considered as a square half-loop antenna with side length $a$ radiating into the groove.

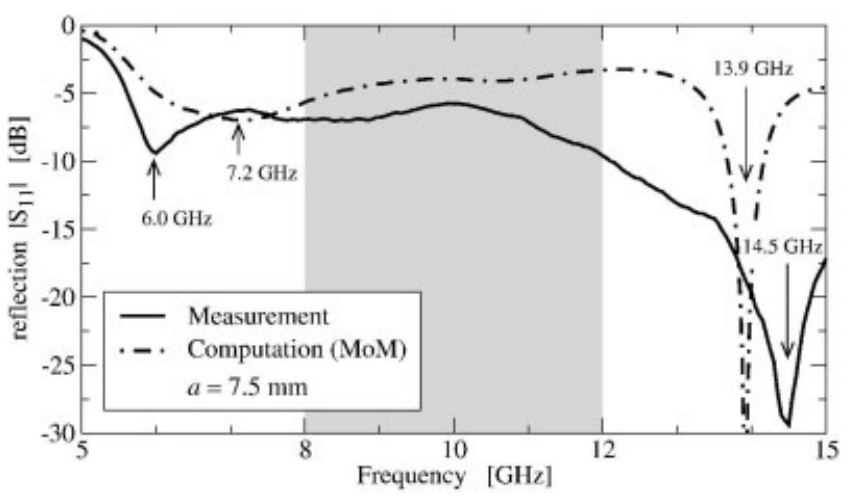

Figure 6 Computed and measured $|S 11(f)|$ of loop: $a=7.5 \mathrm{~mm}$ 


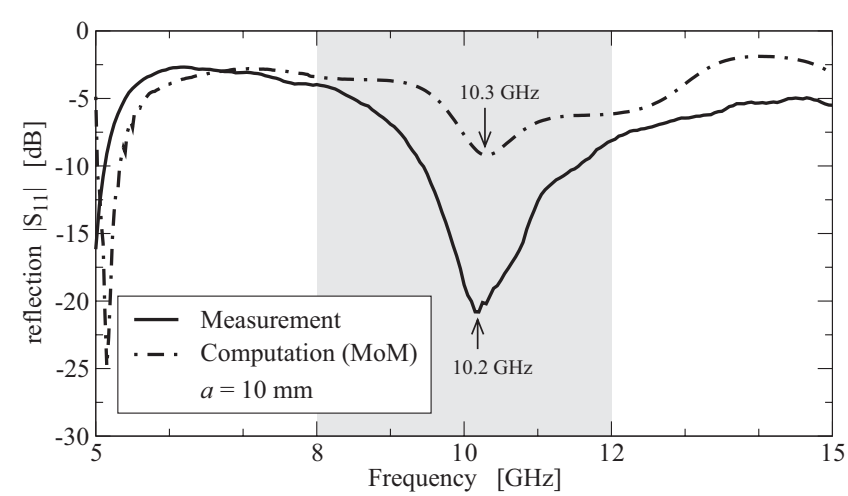

Figure 7 Computed and measured $|S 11(f)|$ of loop: $a=10 \mathrm{~mm}$

The impedance of a circular loop has been calculated in [17] and depends on the wire radius. However, the half-loop antenna is of rectangular shape and affected by the groove structure. The reflection coefficient of the square half-loop antenna inside the groove has been computed by means of the Method of Moments (MoM) for three different loop sizes. For the same loop sizes, the reflection coefficient has been measured. Figures 5-7 compare simulated and measured data. For comparison, the resonant frequencies have also been computed for the square half-loop on infinite ground. The computed and measured resonances are listed in Table 2.

As can be seen from the figures and the table, there is good agreement between simulation of the half-loop in the groove structure and measurements. To use the square loop coupling structure for the X-band, a side length of about $a=5 \mathrm{~mm}$ or $a=$ $10 \mathrm{~mm}$ is suitable.

\subsection{Slot Coupling}

Figure 8 depicts the cross section of the slot with taper length $T$ and width $s$. The tapered region is a radial waveguide of sectoral cross section [18] with the characteristic impedance $Z^{\prime}(r)$. The taper is connected to a rectangular waveguide $\left(\mathrm{TE}_{10}-\right.$ mode) with the impedance $Z$.

The characteristic impedances are given as

$$
Z=\frac{Z_{0}}{\sqrt{1-\left(\begin{array}{c}
\lambda_{0} \\
\lambda_{c}
\end{array}\right)^{2}}}
$$

and

$$
Z^{\prime}(r)=\frac{Z_{0}}{\sqrt{1-\left(\begin{array}{c}
\lambda_{0} \\
\lambda_{c}
\end{array}\right)^{2}}} \cdot \frac{2 \Phi r}{a}
$$

TABLE 2 Computed and Measured Resonances for the Square Loop

\begin{tabular}{cccc}
\hline & \multicolumn{3}{c}{ Loop Resonances in GHz } \\
\cline { 2 - 4 } $\begin{array}{c}\text { Loop Size } \\
a\end{array}$ & $\begin{array}{c}\text { Half-Loop on } \\
\text { Infinite } \\
\text { Ground MoM }\end{array}$ & $\begin{array}{c}\text { Half-Loop Inside } \\
\text { Groove MoM }\end{array}$ & $\begin{array}{c}\text { Half-Loop Inside } \\
\text { Groove } \\
\text { Measurement }\end{array}$ \\
\hline $5.0 \mathrm{~mm}$ & 10.8 & 10.9 & 10.6 \\
$7.5 \mathrm{~mm}$ & 7.1 and 14.4 & 7.2 and 13.9 & 6.0 and 14.5 \\
$10.0 \mathrm{~mm}$ & 5.3 and 10.7 & 5.2 and 10.3 & 5 and 10.2 \\
\hline
\end{tabular}

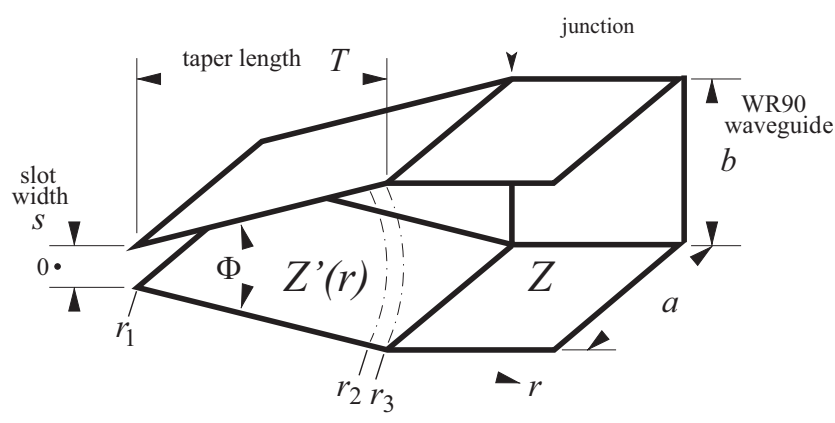

Figure 8 Slot coupling structure with taper length $T$ and width $s$ [see Fig. $1(\mathrm{c})]$

respectively. The width of the waveguides is $a . Z_{0}$ is the intrinsic impedance in free space, and $\lambda_{0}$ and $\lambda_{c}$ are the wavelength in free space and the cut-off wavelength, respectively. The angle $\Phi$ in radian and the radii $r_{1}, r_{2}$, and $r_{3}$ can be calculated by using waveguide height $b$, taper length $T$, and slot width $s$. For minimum reflection the impedances $Z$ and $Z^{\prime}(r)$ must be equal at the junction of the rectangular and radial waveguide. Since the impedance $Z^{\prime}$ inside the radial waveguide depends on $r$, and the impedance $Z$ inside the rectangular waveguide is constant, a matching problem occurs at the junction. For a long taper length, i.e. small angle $\Phi$, the impedance $Z^{\prime}$ changes little in the vicinity of the junction. A detailed investigation of a tapered waveguide is presented in [19]. With the given parameters $s=0.5 \mathrm{~mm}, T=40 \mathrm{~mm}$, and $a$ $\times b=22.86 \times 10.16 \mathrm{~mm}^{2}$ (WR90 standard) the reflection is calculated to be

$$
\Gamma=\left|S_{11}\right|=10 \cdot \log \left|\frac{Z^{\prime}\left(r_{2}\right)-Z}{Z^{\prime}\left(r_{2}\right)+Z}\right|^{2}=-24.7 \mathrm{~dB}
$$

The measured reflection $S_{11}(f)$ is less than $-7 \mathrm{~dB}$ within the $\mathrm{X}$-band as shown in Figure 9. The transition from the rectangular waveguide to the tapered waveguide was fabricated in two pieces. Because of the limited mechanical precision and imperfect alignment of the two waveguides at the junction, the measured reflection is higher than the calculated one but still sufficiently low.

\section{GROOVE GUIDE RESONATOR AND COUPLING STRUCTURES}

In Figure 10, an exploded three-dimensional view of the resonator structure under investigation is depicted. The physical dimensions of the groove structure were given in Section 2, and the resonator length is $L=100 \mathrm{~mm}$. Numerical computations and measurements

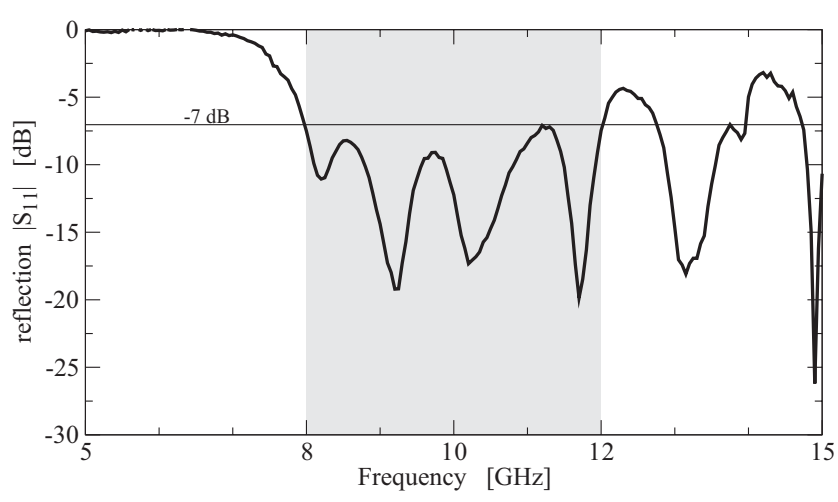

Figure $9|S 11|$ measurement: Characteristic of slot excitation 


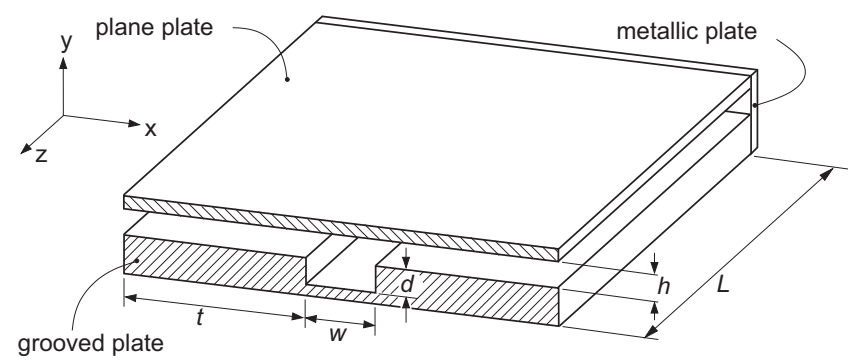

Figure 10 Exploded 3D view of the groove guide resonator [9]

are performed and compared for the three coupling structures placed inside the resonator.

As a versatile tool for solving electromagnetic problems and as a simulation tool, the FDTD method [20] is often used. The computed data here is obtained as in [9]. The groove guide resonator together with its coupling structures is discretized within the Cartesian coordinate system. A cubic cell size of $\sim 0.25 \mathrm{~mm}$ is chosen, which guarantees a sufficiently accurate discretization of the excitation structures. The boundary of the FDTD domain is a 10-cells thick perfectly matched layer (PML). To guarantee stability and low numerical dispersion of the FDTD method, a time step of $\Delta t=0.4815 \mathrm{ps}$ is chosen. The total observation time is set to $16 \mathrm{~ns}$, which corresponds to a frequency resolution of about 60 $\mathrm{MHz}$, after applying an off-line fast Fourier transform.

The source which is implemented in the FDTD algorithm is a modulated Gaussian-function. Its center frequency of maximum amplitude equals $10 \mathrm{GHz}$, and the bandwidth is $10 \mathrm{GHz}$, which covers the X-band. The source is applied as an E-field at one cell inside the coupling structures. For instance [in Fig. 1(c)], the source is centered inside the tapered waveguide and defines the E-field in the $z$-direction.

For each coupling structure, the reflection $\left|S_{11}\right|$ versus frequency was measured by means of a network analyzer. Resonances are at those frequencies where the reflection shows a minimum.

Errors occur in numerical simulations and during the fabrication process and are expected as follows: The frequency resolution in the FDTD simulations was determined to be $60 \mathrm{MHz}$. Since the rectangular groove guide resonator was discretized within the Cartesian coordinate system, there was additionally no discretiza-

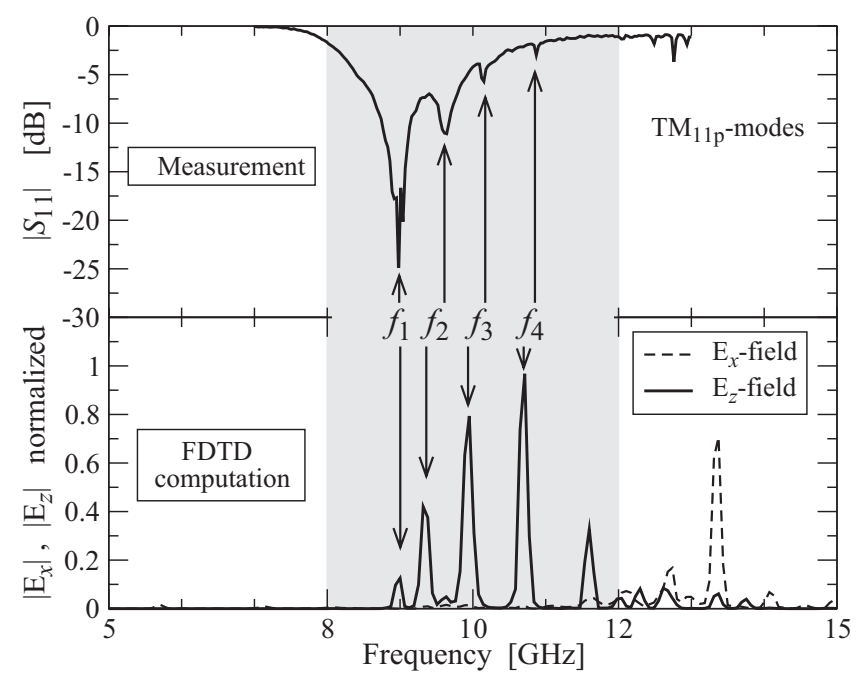

Figure 11 Computed (from [9]) and measured results for the probe
TABLE 3 Simulated and Measured Resonances for the Probe

\begin{tabular}{lccrr}
\hline & \multicolumn{4}{c}{$\mathrm{TM}_{11 \mathrm{p}}$ Resonances in $\mathrm{GHz}$ for the Probe } \\
\cline { 2 - 5 }$p$ & Simulation & Measurement & Error \\
\hline 1 & 9.00 & 8.98 & $-20 \mathrm{MHz}$ & $-0.2 \%$ \\
2 & 9.32 & 9.62 & $+300 \mathrm{MHz}$ & $+3.2 \%$ \\
3 & 9.95 & 10.12 & $+170 \mathrm{MHz}$ & $+1.7 \%$ \\
4 & 10.71 & 10.87 & $+160 \mathrm{MHz}$ & $+1.5 \%$ \\
\hline
\end{tabular}

tion error. The mechanical tolerances of the fabricated resonator structure are $\pm 0.1 \mathrm{~mm}$. This corresponds to a deviation of the resonance frequencies of about $\pm 50 \mathrm{MHz}$, whereas plate distance $h$ and groove depth $d$ are the most critical parameters.

\subsection{Probe Coupling}

Figure 11 shows the simulation results together with the measurement result for the probe. According to Section 2.1, the probe length is chosen as $l=24 \mathrm{~mm}$. The resonances are noted with $f_{1}$ to $f_{4}$. Since the Ey-field is much smaller than the other components, it is not shown in Figure 11. When the $x$ - and $z$-components of the E-field in the figure are compared, it is seen that the $E_{\mathrm{z}}$-field is stronger than the $E_{\mathrm{x}}$-field and exhibits clear resonances within the X-band. Therefore, the probe mounted as shown in Figure 1 can be assumed to excite TM-modes. This result can also be concluded by inspecting the mode patterns depicted in $[2,8]$. Table 3 lists simulated and measured values of the resonance frequencies together with errors. The simulated values show good agreement with the measured ones with a small error.

\subsection{Loop Coupling}

For the investigation of the loop coupling a loop size of $A$ $=10 \times 10 \mathrm{~mm}^{2}$ chosen according to Section 2.2. Simulation and measurement results are shown in Figure 12. Reducing the plate distance $h$ causes the resonances to shift to higher frequencies. The resonances noted with $f_{1}$ to $f_{6}$ are identified by changing the plate distance. Since the loop excites TE-modes, and the $y$-component of the electric field is much smaller in magnitude, only the simulated Ex-field is plotted. Table 4 lists simulated and measured values of the resonance frequencies together with errors. The simulated values for the loop also show good agreement with the measured ones with a small error, as in the probe case.

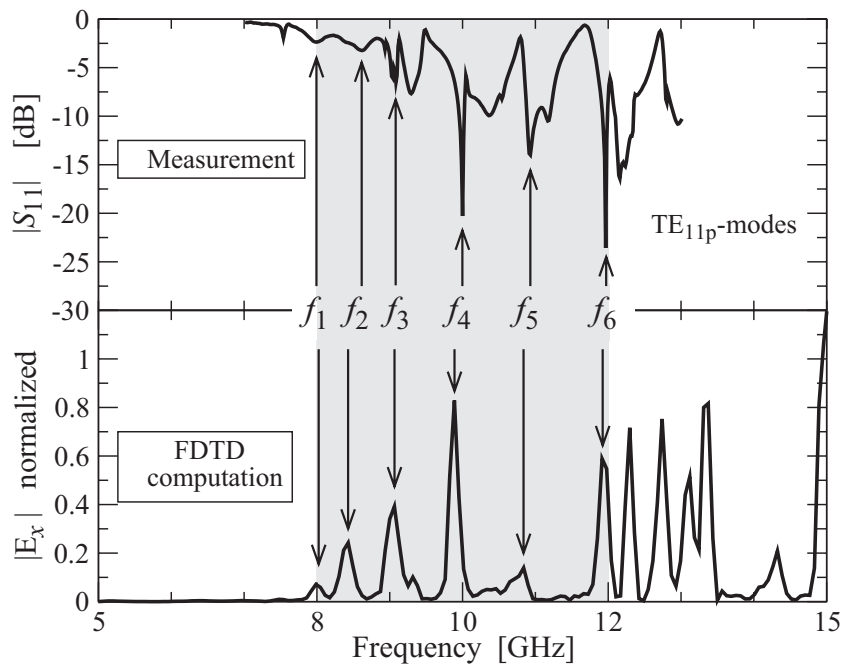

Figure 12 Computed [9] and measured results for the loop 


\begin{tabular}{lcccr} 
& \multicolumn{3}{c}{$\mathrm{TE}_{11 \mathrm{p}}$ Resonances in $\mathrm{GHz}$ for the Loop } \\
\cline { 2 - 5 }$p$ & Simulation & Measurement & $0.0 \%$ \\
\hline 1 & 7.99 & 7.99 & $0 \mathrm{MHz}$ & $0.0 \%$ \\
2 & 8.43 & 8.62 & $+190 \mathrm{MHz}$ & $+2.2 \%$ \\
3 & 9.06 & 9.04 & $-20 \mathrm{MHz}$ & $-0.2 \%$ \\
4 & 9.89 & 10.00 & $+110 \mathrm{MHz}$ & $+1.1 \%$ \\
5 & 10.84 & 10.93 & $+90 \mathrm{MHz}$ & $+0.8 \%$ \\
6 & 11.92 & 11.96 & $-40 \mathrm{MHz}$ & $-0.3 \%$ \\
\hline
\end{tabular}

\subsection{Slot Coupling}

The groove guide resonator can also be excited using the slot coupling structure given in Section 2.3. Simulation and measurement results are shown in Figure 13. The slot in this work is oriented in such way that it excites TM-modes and only the simulated $E_{\mathrm{z}}$-field is plotted. Table 5 lists simulated and measured values of the resonance frequencies. In terms of frequency deviation, the results for the slot coupling, compared with the other two coupling structures, shows the best agreement between simulation and measurement.

\section{CONCLUSION}

As an alternative to the classical mode launcher for groove guide structures, three types of coupling structures have been examined, viz. a probe, loop, and slot. Probe and loop show resonance characteristics and were optimized to operate in the X-band. The frequency error between measurement and computation for various coupling structure dimensions was less than $3 \%$ for the probe and around $10 \%$ for the loop. The slot operates above cut-off and shows a reflection of less than $-7 \mathrm{~dB}$. The designed probe and slot excite TM-modes, and the loop excites TE-modes.

Based on the results obtained from the coupling structure examinations, a groove guide resonator with the same coupling structures was investigated. For numerical computations, the FDTD method was used. The resonator with its coupling structures was fabricated and resonance frequencies were determined by measuring the reflection coefficient. With a maximum error of $3.2 \%$ for the probe and loop, the measurement results are in good agreement with the computed data. Best results were obtained for the slot, with an error of less than $0.4 \%$.

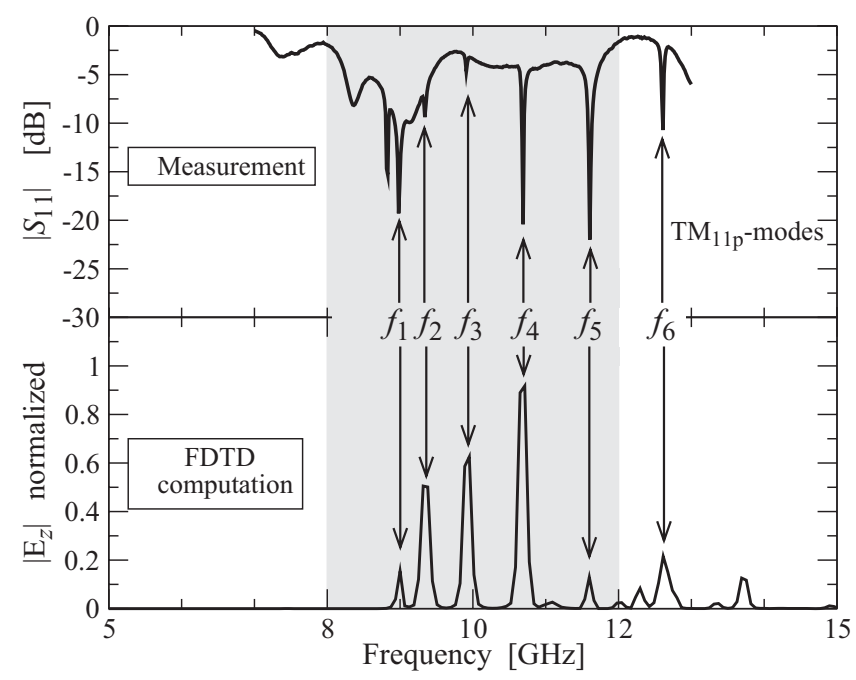

Figure 13 Computed [9] and measured results for the slot

\begin{tabular}{lcccr}
\hline & \multicolumn{3}{c}{$\mathrm{TM}_{11 \mathrm{p}}$ Resonances in $\mathrm{GHz}$ for the Slot } \\
\cline { 2 - 5 }$P$ & Simulation & Measurement & \multicolumn{2}{c}{ Error } \\
\hline 1 & 9.00 & 9.98 & $-20 \mathrm{MHz}$ & $-0.2 \%$ \\
2 & 9.32 & 9.34 & $+20 \mathrm{MHz}$ & $+0.2 \%$ \\
3 & 9.95 & 9.91 & $-40 \mathrm{MHz}$ & $-0.4 \%$ \\
4 & 10.71 & 10.69 & $-20 \mathrm{MHz}$ & $-0.2 \%$ \\
5 & 11.60 & 11.60 & $0 \mathrm{MHz}$ & $0.0 \%$ \\
6 & 12.61 & 12.61 & $0 \mathrm{MHz}$ & $0.0 \%$ \\
\hline
\end{tabular}

\section{ACKNOWLEDGMENT}

The authors would like to thank the Scientific and Technical Research Council of Turkey (TUBITAK) for its financial support of the project.

\section{REFERENCES}

1. F.J. Tischer, The groove guide, a low-loss waveguide for millimeter waves, IEEE Trans Microwave Theory Tech 11 (1963), 291-296.

2. T. Nakahara and N. Kurauchi, Transmission modes in the grooved guide, in SUMITOMO, Electron Tech Rev 5 (1965), 65-71.

3. A.A. Vertiy, S.P. Gavrilov, S. Aydinlik, and S. Samedov, Circular groove shaped resonator for millimeter waves, Int J Infrared Millimeter Waves 17 (1996), 1613-1637.

4. A.S. Aydinlik Bechteler and L. Sevgi, Millimeter waveband semisymmetrical groove guide resonators, IEEE Microwave Mag 5 (2004), 51-60.

5. Y.M. Choi, Novel method of measuring dielectric properties at 100 GHz using a groove-guide resonator, IEE Proc Microwave Antennas Propagat 135 (1988), 138-140.

6. Z. Ma and E. Yamashita, A new method for the characterization of groove-guide leaky-wave antenna with an asymmetrically located metal strip, IEEE Microwave Guided Wave Lett 2 (1992), 489-491.

7. Y. Liu and H.-S. Yang, Wide-band circular groove guide horn antenna, IEEE Trans Antennas Propagat 51 (2003), 395-398.

8. J.W.E. Griemsmann, The groove guide, the symposium on quasioptics, Polytechnic Inst., Brooklyn, 1964, pp. 565-578.

9. Th.F. Bechteler, Analysis of excitations for a groove guide resonator at $10 \mathrm{GHz}$ by means of the FDTD method, Int J Infrared Millimeter Waves 26 (2005), 819-830.

10. M. Fernyhough and D.V. Evans, Full multimodal analysis of an open rectangular groove waveguide, IEEE Trans Microwave Theory Tech 46 (1998), 97-107.

11. C.A. Balanis, Antenna theory-Analysis and design, Wiley, New York, 1997.

12. J.D. Kraus and R.J. Marhefka, Antennas for all applications, McGrawHill, New York, 2002

13. G.H. Brown and R. King, High-frequency models in antenna investigations, Proc IRE 22 (1934), 457-480.

14. C.T. Tai, Coupled antennas, Proc IRE 36 (1948), 487-500.

15. M. Abramovitz and I.A. Stegun, Handbook of mathematical functions, Dover, New York, 1970.

16. R.F. Harrington, Field computation by moment methods, IEEE Press, New York, 1993.

17. G. Zhou, and G.S. Smith, An accurate theoretical model for the thin-wire circular half-loop antenna, IEEE Trans Antennas Propagat 39 (1991), 1167-1177.

18. N. Marcuvitz, Waveguide handbook, IEE Electromagn Wave Series 21, Peter Peregrinus Ltd., Exeter, 1993.

19. S. Dwari, A. Chakraborty, and S. Sanyal, Analysis of linear tapered waveguide by two approaches, Prog Electromagn Res PIER 64 (2006) 219-238.

20. A. Taflove, Computational electrodynamics-The finite-difference time-domain method, Artech House, Norwood, 1995. 